\title{
Rapid clump formation in discs of young stellar objects class O-I
}

\author{
Olga P. Stoyanovskaya ${ }^{1,2}$ and Valeriy N. Snytnikov ${ }^{1,2}$ \\ ${ }^{1}$ Boreskov Institute of Catalysis SB RAS \\ Pr. Academika Lavrentieva, 5, 630090, Novosibirsk, Russia \\ email: stop@catalysis.ru, snyt@catalysis.ru \\ ${ }^{2}$ Novosibirsk State University, \\ Str. Pirogova, 2, 630090, Novosibirsk, Russia
}

\begin{abstract}
The formation of planetesimals and large bodies in circumstellar discs can be referred to the stage of massive unstable disc emerging due to the gravitational collapse in the molecular cloud. We have simulated the dynamics of gas and boulder clumps in such systems. The features of instability development in massive disc is discussed. Unlike medium-massive discs the massive disc can provide conditions for simultaneous formation of several clumps as a result of overdensity ring fragmentation. We found regimes where 3,2 or 1 overdensity ring appear in the disc and then fragment into clumps collecting about a half of the disc mass.
\end{abstract}

Keywords. circumstellar matter, Young Stellar Object (YSO) class O-I, gravitational instability, self-gravitating clump formation.

Circumstellar discs of young stellar objects of class O-I can reach the threshold of gravitational instability development leading to the appearance of solitary overdensity areas or clumps. Such clumps may play a critical role in planetary system formation, being embryos of gas giant planets or brown dwarfs, collecting solids for planetesimal and large body formation. Numerical modeling of gravitational instability development demonstrates that depending on disc and central body parameters the clumps can be formed in radial fragmentation of global overdensity rings or in spiral sleeves of different nature.

In low- and medium-mass discs with intense cooling, clumps are formed in spiral arms; the first clump first appears and then affects the gas flow in the disc, which may provide the possibility of clump formation at a smaller radius Helled et al. (2014). For a cold massive disc, the isolated high-density regions are formed by fragmentation of a dense ring emerging due to development of the global Toomre instability. Unlike the case of medium- and low-mass disc, the formation of several rings in a massive disc allows the fragmentation to start not at a maximum radius (see Fig.1). Fig.1 shows the logarithm of the gas surface density during an orbital period.

In this study we investigate the scenario where rings are initial stage of gravitational instability development. Computational experiments were carried out within a quasi-3D model of the disc described in Snytnikov \& Stoyanovskaya (2013). The hybrid model of massive disc includes Eulear equations for gas dynamics with enthropy formulation of energy equation, collisionless Boltzmann equation for $1 \mathrm{up}$ to $10 \mathrm{~m}$ in size primary bodies dynamics, and Poisson equation for self-consistent gravitational field. The numerical model is based on SPH and PIC methods combined with grid method for Poisson equation solving.

In our simulation of massive disc dynamics we found regimes where 3,2 or 1 overdensity rings fragment into clumps. The total mass of clumps can reach up to half mass of the 

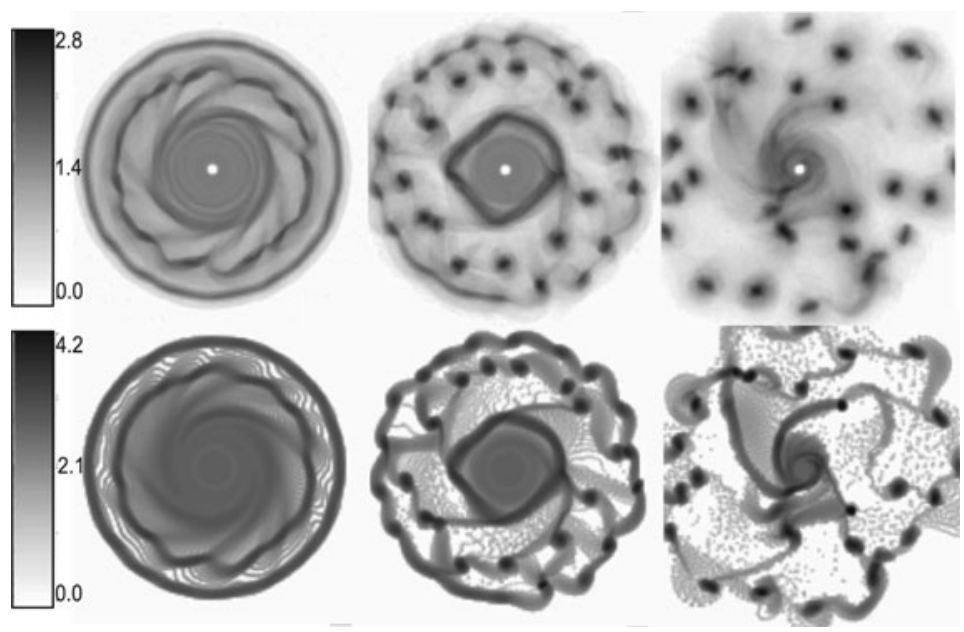

Figure 1. The surface density logarithm of the gas (bottom) and the primary solids subdisc (top) for time points $\mathrm{T}=7.5,10$, and 12.5. The orbital time of the outer part of the disc is 15. The disc has a radius of $20 \mathrm{AU}$, the central body had mass $M_{c}=0.45 M_{0}$, and the disc was represented by a gas component with mass $M_{g a s}=0.52 M_{0}$ and a primary solids subdisc with $M_{\text {par }}=0.03 M_{0}$. The effective adiabatic exponent was $\gamma=5 / 3$, initial gas temperature at $R=10$ au was $235 \mathrm{~K}$, initial velocity dispersion of solids was $95 \mathrm{~m} \mathrm{~s}^{-1}$. The numerical resolution of the calculation was $16 * 10^{4} \mathrm{SPH}$ particles, $10^{7} \mathrm{PIC}$ particles, $\frac{h_{r}}{R}=0.01$.

disc. The minimum found value of ratio of fragmenting ring radius to the disc radius was 0.25 for polynomial density distribution and 0.1 for exponential density distribution. Varying the central body mass we found its effect on fragmentation order of the rings: the ring of smaller radius becomes more stabilized against fragmentation by the increasing of the central mass. During the rings fragmentation simultaneous formation of several high-density areas of gas and solids at almost the same radius takes place. In a massive disc, the emerging clumps are closely surrounded by neighbour clumps, and they can move from the centre to the periphery over short time periods under the action of the gravitational fields of other regions.

We have demonstrated that the gravitational field of the isolated high-density areas could capture bodies from 1 up to $10 \mathrm{~m}$ in size (without regard to the frictional force between the gas and such bodies) to form epicyclic trajectories both in the initial stages of collapse and before collapse. Triggering a gravitational collapse in such objects (highdensity areas) can transform them into planet embryos or into regions of intense growth of solids. A key problem here is to estimate the typical lifetime of such objects and to determine whether they can exist for a time period sufficient for launching a mechanism that moves the disc to the next stage of planetary formation.

This work was supported by RFBR project 14-01-31516, the RAS Presidium programs 'Biosphere origin and evolution' and 'Origin, structure and evolution of objects in the Universe', as well as by the SB RAS Integration Project N130.

\section{References}

Snytnikov, V. N. \& Stoyanovskaya, O. P., 2013, MNRAS, 428, 2

Helled, R., Bodenheimer, P., Podolak, M., Boley, A., Meru, F., Nayakshin, S., Fortney, J. J., Mayer, L., Alibert, Y., \& Boss, A. P. Giant Planet Formation, Evolution, and Internal Structure, in Protostar and Planets VI, arXiv:1311.1142v1 